\title{
Discursos, prácticas y temáticas de maestros y maestras en primera infancia acerca de la diversidad sexual y de género: límites y posibilidades para la consolidación de una educación inclusiva
}

Fidel Mauricio Ramírez Aristizábal

Jeison Andrés Cardona Zuluaga ${ }^{2}$

Recibido: 05-05-2020

Aceptado: 16-06-2020

\section{Resumen}

Desde inicios del presente siglo se han dado importantes desarrollos jurídicos y políticos para promover el reconocimiento, respeto y valoración de la diversidad sexual y de género; no obstante, estos esfuerzos han sido insuficientes para transformar la realidad de discriminación y violencia que enfrentan día a día estos sectores poblacionales en los ambientes escolares, lo cual se debe, principalmente, a la indiferencia con que maestros y maestras asumen este tema o a sus propios prejuicios ideológicos. Tal situación es puesta en evidencia en el presente artículo,

1. Doctor y magíster en Educación, Línea de Educación, Derechos Humanos y Ciudadanías licenciado en Filosofía, Pensamiento Político y Económico. Docente e investigador, Universidad El Bosque.

Correo electrónico: fmramirez@unbosque.edu.co; fidel.ramirez1281@gmail.com Google Scholar: https://scholar.google.com/citations?user=enR571EAAAAJ\&hl=es ORCID: https://orcid.org/0000-0003-1210-5128

CvLac: http://scienti.colciencias.gov.co:8081/cvlac/visualizador/generarCurriculoCv.do?cod $\mathrm{rh}=0001406480$

2. Magíster y especialista en Educación; licenciado en Filosofía y Letras. Docente e investigador, Universidad Santo Tomás.

Correo electrónico: jeisoncardona@usantotomas.edu.co; jeison1803@gmail.com

Google Scholar: https://scholar.google.com/citations?user=OPt7S4YAAAAJ\&hl=es

ORCID: https://orcid.org/0000-0002-9786-5144

CvLac: http://scienti.colciencias.gov.co:8081/cvlac/visualizador/generarCurriculoCv.do?cod $\mathrm{rh}=0001499440$ 
producto del ejercicio investigativo autoetnográfico realizado durante un proceso de formación a docentes de primera infancia de diferentes secretarías de educación a nivel nacional. Con este proceso se espera sensibilizar sobre la urgente necesidad de trabajar para formar en la construcción de ciudadanías sexuales desde el escenario educativo.

Palabras clave: autoetnografía, diversidad sexual y de género, inclusión educativa, discursos, prácticas y temáticas docentes.

\section{Discourses, practices and themes of early childhood teachers about sexual and gender diversity: limits and possibilities for the consolidation of an inclusive education}

\section{Abstract}

Since the beginning of the present century, relevant legal and political developments have been taking place that promotes the recognition, respect, and valuation of sexual and gender diversity; However, these efforts have been insufficient to transform the reality of the discrimination and violence that these days will face in these population sectors in school settings; this, mainly, by the indifference with the teachers assume this subject or their ideological prejudices. A situation that is highlighted in this article as a result of the autoethnographic investigative exercise carried out during a training process for early childhood teachers from different Secretariats of Education at the national level. With this work, we hope to raise awareness of the urgent need to work to train teachers around the construction of sexual citizenships from the educational setting.

Keywords: Autoethnography, sexual and gender diversity, educational inclusion, discourses, practices and teaching themes. 


\section{Introducción}

De las manifestaciones en Stonewall ${ }^{3}$ a la declaración de la Corte Interamericana de Derechos Humanos a favor del reconocimiento del matrimonio igualitario en 2018 han transcurrido casi cincuenta años; tiempo en el cual se dio paso - al menos en los discursos oficiales- de la patologización o penalización de la conducta homosexual, al reconocimiento, respeto y protección de las personas con orientaciones sexuales e identidades de género diversas; asociaciones médicas coinciden al señalar que la homosexualidad es una característica que no puede ser considerada como enfermedad o un desorden psicoemocional que revista algún tipo de riesgo para la vida y el normal desarrollo de las personas (APA, 2002); a nivel jurídico se logró la despenalización de la homosexualidad en casi todos los países occidentales y se han desarrollado políticas que buscan la protección y superación de los prejuicios en contra de las personas LGBT. De hecho, en países de Oriente se empieza a avanzar a este respecto, como es el caso de la India, que en 2018, después de 157 años, despenalizó la homosexualidad (BBC, 6 de septiembre de 2018).

A nivel educativo el asunto es más complejo, y se ha convertido en un campo de tensión en el que se encuentran enfrentados quienes consideran que en la escuela es necesario incorporar discursos, prácticas y temáticas que favorezcan el reconocimiento, respeto y valoración de la diversidad sexual y de género; y quienes consideran que este tipo de acciones son una estrategia de imposición de la homo- y la transexualidad a niños, niñas y jóvenes; en los últimos años este conflicto ha cobrado gran importancia y se ha convertido en un factor decisivo incluso en los procesos electorales llevados a cabo en la región latinoamericana.

\footnotetext{
3. Stonewall se ha convertido en un hito del movimiento de reivindicación de los derechos de las personas LGBT; se trata de la conmemoración de los hechos acontecidos en la madrugada del 28 de junio de 1969, en el pub conocido como Stonewall Inn, ubicado en un barrio neoyorquino, cuando un grupo de trans decidió reaccionar ante los continuos hostigamientos de la policía debido a su expresión de género (Armstrong y Crage, 2006).
} 
Desde mediados de 2012, en países de Europa y América algunos grupos antiderechos ${ }^{4}$ popularizaron la idea de la existencia de una estrategia de los sectores LGBT para imponer una cultura homosexual; bajo la idea de una "ideología de género", estos grupos lograron revivir prejuicios religiosos para oponerse a los avances en materia de derechos civiles de personas con orientaciones sexuales e identidades de género no hegemónicas (Mena-López y Ramírez, 2018). Más que una verdadera preocupación por la protección de las familias, de los niños y niñas, es una apuesta de sectores económicos neoliberales para hacer retroceder los avances sociales a favor de personas que tradicionalmente han sido excluidas, y recobrar el control político para imponer sus agendas. Estos grupos crearon un fantasma para generar miedo (Vilà, 2016).

En Colombia, durante 2016, con el proyecto de renovación de los manuales de convivencia escolar para suprimir distintas formas de discriminación y violencia homofóbica y transfóbica que se legitimaban a través de estos, se encontró el ambiente propicio para generar pánico en torno a la posibilidad de que reconocer los derechos de estudiantes LGBT se convirtiera en un escenario propicio para la confusión sexual y de género de los demás estudiantes.

Las cartillas que el Ministerio de Educación Nacional creó, con asesoría de organismos educativos internacionales, para sensibilizar a las comunidades educativas en contra de la violencia homofóbica y transfóbica fueron presentadas al público incauto como si fueran verdaderos materiales pornográficos preparados para corromper la conciencia de los niños y niñas, lo cual hicieron a través de publicidad falsa (El Espectador, 8 de agosto de 2016); los materiales generaron gran indignación entre los padres de familia y rápidamente los líderes religiosos tomaron la vocería en nombre de cristianos y cristianas para oponerse a lo que llamaron: una estrategia en contra de la familia, donde los homosexuales querían imponer su ideología de género.

4. Se asume el calificativo antiderechos para hacer referencia a los grupos que se oponen a los avances en materia de derechos de personas LGBT y a la despenalización del aborto. Este calificativo remplaza el de provida, con el que se han autodenominado los miembros de estos grupos, escondiendo su trasfondo ideológico que se constituye en un ataque a los derechos humanos. 
Al lado del cubrimiento mediático que tuvieron las denuncias contra la llamada "ideología de género" se hizo un llamado a organizaciones de padres de familia, comunidades e iglesias; la invitación tuvo una importante acogida y lograron movilizar miles de personas; no obstante, la atención que se había puesto a las cartillas se desplazó a otros lugares, como el acuerdo que se estaba negociando por esos días en La Habana entre la guerrilla de las FARC (Fuerzas Armadas Revolucionarias de Colombia) y el Gobierno nacional, que de un momento a otro también entró a hacer parte del debate por "estar infestado" de una ideología de género (Semana, 9 de septiembre de 2016), en este caso debido a que en los acuerdos se había incorporado un enfoque de género para el tratamiento de las víctimas en el marco de violencia armada. Como consecuencia, ni la renovación de los manuales de convivencia escolar, ni el acuerdo de La Habana se han podido implementar adecuadamente.

La situación, desde ese momento y hasta ahora, se ha tornado complicada para personas con orientaciones sexuales e identidades de género diversas; con el reposicionamiento de discursos de odio se han obstaculizado los procesos de implementación de las estrategias que por ley se habían definido para lograr superar las barreras que se les imponen día a día en el sistema educativo a estudiantes LGBT y disminuir la discriminación y violencia que viven estos sectores en distintos estamentos sociales. Se ha generado una paranoia social en torno a la necesidad y pertinencia de los derechos humanos de ciertos sectores, no solamente el LGBT, sino que también los derechos de la mujer, el migrante y el ambiente se han visto cuestionados.

No obstante, ha sido difícil lograr un diagnóstico que permita dimensionar el impacto de la estrategia antiderechos que se orquestó en contra de la revisión de los manuales de convivencia y el establecimiento de estrategias conducentes a atender, mitigar y prevenir la homofobia y la transfobia en los sistemas educativos. Razón por la cual, dada la posibilidad de recorrer varias secretarías de educación del país en el marco de un proceso de formación dirigido a docentes de primera infancia con respecto al Decreto 
1421 de 2017, se desarrolló la investigación que buscó responder a la pregunta acerca de cuáles son los límites y las posibilidades de una educación inclusiva que se derivan del análisis de discursos, prácticas y temáticas de maestros y maestras de primera infancia en torno a la diversidad sexual y de género, ejercicio que se realizó desde una perspectiva autoetnográfica (Blanco, 2012).

Por otro lado, aunque se reconocen las lógicas detractoras de este tipo de estudios, se considera que dada la situación esta metodología es la más apropiada, porque este tipo de investigación es la que incorpora elementos de la actividad etnográfica y la biografía de los investigadores; con lo cual se enfatiza en el análisis cultural y la interpretación de sus comportamientos, desde sus pensamientos y experiencias; habitualmente a partir del trabajo de campo, en relación con los otros y con la sociedad que estudia; o sea, la autoetnografía explora la interacción entre el yo personal y la dimensión social de los sujetos que se interrelacionan (Panourgia, 1997).

En tal sentido, más que la contundencia de la información en torno a discursos, prácticas y temáticas de maestros y maestras de primera infancia acerca de la diversidad sexual y de género, el aporte de este trabajo apunta a la exposición de la perspectiva de los autores desde unos referentes conceptuales provenientes de los estudios de género, para interpretar lo vivido en los diversos espacios de interacción de dichos maestros. Es decir que hay una relación constante entre la teorización y las cotidianidades de los sujetos.

\section{Metodología}

Desde que Karl G. Heider (1975) y David Hayano (1979) propusieron el término autoetnografía, se ha dado lugar a diversas experiencias de investigación que la utilizan como un recurso metodológico y epistemológico de gran valor para las ciencias sociales, por recurrir a la utilización de lo autobiográfico como materia prima, a partir de 
la cual el etnógrafo se sumerge en la propia experiencia personal y cultural en torno a un tema particular de estudio (Atkinson, 2013).

Los diseños autoetnográficos son guiados por la lógica narrativa (Guerrero, 2014), en la que se une el sujeto (investigador) y el objeto (su experiencia); esto genera al final una suerte de autoconciencia y autodescubrimiento, no solo sobre sí mismo, sino también de la realidad social y del contexto histórico-cultural en el cual se desarrolla la práctica investigativa (Guerrero, 2016). Este proceso inicia con una introsprección-reflexiva, donde la ecuación personal juega un papel decisivo en la comprensión de los fenómenos y realidades estudiadas; del horizonte de sentido propio (Banguero Camacho, 2007) se desprende el interés particular y la construcción teórica.

El etnógrafo explora su mundo y lo hace al momento de develar las conexiones entre lo personal y lo cultural, con lo cual aporta un profundo sentido antropológico a sus actos, emociones, creencias y vivencias. La autoetnografía, como método de investigación, proporciona un acceso privilegiado al mundo personal del investigador, en el cual puede recrear un mapa o cartografía de referencias biográficas insertadas en el entorno sociocultural del que forma parte, y de donde nacen esas mismas referencias para, finalmente, crear una historia, una trama o argumento en el cual quedan entrelazados los hechos y experiencias biográficas. En ese proceso particular de narrar o contar la propia historia, el investigador-narrador adopta unas veces la perspectiva de un insider al tiempo que dirige la mirada y focaliza su atención hacia la realidad cultural como un outsider (Panourgia, 2000).

De acuerdo con lo anterior, esta investigación se desarrolló en tres etapas: 1) la primera de ellas destinada a la revisión conceptual, construida en torno a la diversidad sexual y de género, a lo largo del proceso investigativo en esta materia; 2 ) en la segunda etapa se realizó un proceso de observación de los discursos, prácticas y temáticas de docentes de primera infancia en torno a la diversidad sexual y de género, para lo que se echó mano de instrumentos tales como el diario de campo (García Gómez, 2014) y 
análisis del discurso a los trabajos realizados por los docentes en los espacios de formación y durante las visitas in situ; 3) finalmente, se pusieron en diálogo la experiencia de los investigadores, la teoría en torno a la diversidad sexual y de género en la escuela y los datos recolectados durante el trabajo de campo.

Las categorías centrales de análisis están relacionadas con los discursos, las prácticas y las temáticas relacionadas con la diversidad sexual y de género, las cuales se codificaron como lo muestra la tabla 1.

Tabla 1. Codificación de las categorías de análisis.

\begin{tabular}{|l|l|l|}
\hline \multicolumn{1}{|c|}{ Categoría } & \multicolumn{1}{|c|}{ Subcategoría } & \multicolumn{1}{c|}{ Código } \\
\hline \multirow{2}{*}{$\begin{array}{l}\text { Discursos sobre la } \\
\text { diversidad sexual }\end{array}$} & Conceptos & Con.DSG \\
\cline { 2 - 3 } & Creencias & Cre.DSG \\
\cline { 2 - 3 } & Expresiones & Expre.DSG \\
\hline \multirow{3}{*}{$\begin{array}{l}\text { Prácticas sobre la } \\
\text { diversidad sexual y de } \\
\text { género }\end{array}$} & Experiencias en el quehacer docente & Ex_doc \\
\cline { 2 - 3 } & Experiencias personales & Ex_per \\
\cline { 2 - 3 } & Experiencias ajenas & Ex_aj \\
\hline \multirow{3}{*}{$\begin{array}{l}\text { Temas en torno a la } \\
\text { diversidad sexual y de } \\
\text { género }\end{array}$} & Favorables & Tem_fav \\
\cline { 2 - 3 } & Desfavorable & Tem_des \\
\cline { 2 - 3 } & Neutro & T_neu \\
\hline
\end{tabular}

Fuente: informe de investigación propio.

Aunque se entiende, tal como lo hacen Urra, Muñoz y Peña, que "un discurso se refiere al uso del lenguaje de individuos relacionado a la formación cultural, social y política determinadas por sus interacciones dentro de la sociedad" (2013, p. 53) —o sea, que los discursos traen implícitas las prácticas-, para el caso de esta investigación se hace una separación entre discursos y prácticas, donde los primeros hace referencia a lo que se dice y las prácticas a las acciones que se realizan; por su parte, los temas se refieren a las temáticas abordadas en clase como parte del currículo explícito y oculto. 
Cada una de estas categorías fue analizada a la luz de los límites y las posibilidades para una educación inclusiva, que fue el foco de estudio de esta investigación, como se muestra en la figura 1.

Figura 1. Análisis de categorías.

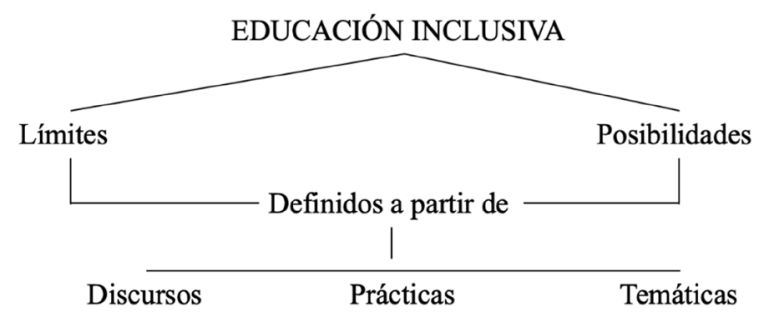

Fuente: construcción propia.

\section{Perspectiva teórica y posicionamiento político de la investigación}

Dada la carga ideológica que esconden muchos estudios de esta naturaleza es importante declarar los marcos teóricos y el posicionamiento político de los autores, con el fin de brindar un horizonte de sentido desde el cual debe ser leído este trabajo. Voluntariamente, se omite un debate acerca del origen de la homosexualidad, por considerarse improductivo, dada la cantidad de perspectivas que existen al respecto y que muchas de ellas son irreconciliables entre sí; se entiende la diversidad sexual como una expresión natural de la diversidad humana y se asume que la valoración negativa que se ha tenido acerca de la homosexualidad, así como de otras expresiones de la sexualidad no conducentes a la procreación, corresponden más a lógicas de poder promovidas en momentos concretos de la historia - con el fin de reprimir y controlar al ser humano e instrumentalizarlo en función de la producción y la perpetuación de desigualdades - que a una realidad cultural e histórica permanente, como lo expone Foucault (2007) en su trabajo sobre historia de la sexualidad. 
Desde la anterior perspectiva, se presenta el marco conceptual bajo el cual se desarrolló la investigación, y además se muestra un recorrido teórico en torno a la diversidad sexual y de género en la escuela. En primer lugar se plantea lo polisémico y complejo del uso de la categoría "diversidad sexual"; después se dejan ver las definiciones de orientación sexual, identidad de género, expresión de género; para finalizar con identidad LGBT.

En lo relacionado al posicionamiento político, se declara que hay una apuesta por el enfoque de derechos humanos, que promueve el reconocimiento, respeto y valoración de las diferentes expresiones de la orientación sexual y la identidad de género, así como todas las particularidades de las cuales se habla actualmente.

\section{Clarificación conceptual}

- Diversidad sexual: es el concepto a través del cual se busca reconocer que la sexualidad tiene múltiples expresiones; ha ganado popularidad como manera de simplificar la sigla LGBT, que con el paso de los años se ha ampliado cada vez más; así mismo, algunos consideran que a través de esta categoría se rompe la dicotomía entre la sexualidad hegemónica y las sexualidades periféricas (Fonseca Hernández y Quintero Soto, 2009). Sin embargo, algunos teóricos y movimientos sociales la consideran perjudicial, pues su uso es un eufemismo que invisibiliza las particularidades de cada grupo poblacional y las demandas que estos tienen para la sociedad, lo que le hace perder la carga política y transgresora de palabras como gay, lesbiana, bisexual o trans. Al respecto, Guillermo Núñez realiza un interesante recorrido que pone en evidencia las posibilidades y los límites de este concepto (Núñez, 2016). En esa obra se utiliza el concepto como forma para referirse a las personas LGBT, expresión que también se utilizó a lo largo de este trabajo. 
- Orientación sexual: la capacidad de una persona para sentir una atracción sexual profunda y emocional hacia otra y tener relaciones sexuales con individuos de un género diferente, del mismo género o de más de un género. Por ejemplo, los hombres gais experimentan atracción sexual hacia otros hombres y la capacidad de tener una relación íntima principalmente con ellos. Las mujeres lesbianas sienten atracción sexual hacia otras mujeres y tienen la capacidad de tener una relación íntima principalmente con ellas. Las personas bisexuales sienten atracción hacia ambos, hombres y mujeres.

- Identidad de género: la experiencia individual e interna de género de una persona sentida íntimamente, que puede corresponder o no al sexo asignado al nacimiento. Esto incluye el sentido personal del cuerpo (que puede involucrar, por libre opción, modificación a la apariencia o función de su cuerpo por medios médicos, quirúrgicos u otros medios) y otras expresiones de género, incluyendo el vestuario, la forma de hablar y los gestos.

- Expresión de género: es la manera como una persona le expresa su propio género al mundo; por ejemplo, a través de nombres, ropa, forma de caminar, modo de hablar, de comunicar, de asumir roles sociales y de su comportamiento en general.

- Identidad LGBT: es la experiencia de identidad y pertenencia como persona diversa al sector de lesbianas, gais, bisexuales y trans, lo que hace de estos grupos verdaderos movimientos sociales que han logrado un importante posicionamiento en materia de derechos. 


\section{Marco teórico}

En la escuela se guarda silencio acerca de la diversidad sexual y de género, tal y como reconoce el documento Diagnóstico de situación de niñas, niños y adolescentes con orientaciones sexuales o identidades de género no normativas: "[...] la situación actual de la niñez no normativa en Bogotá, [es un] tema que muchas veces no se toca porque socialmente se invisibiliza la sexualidad en algunas etapas vitales [...]" (Alcaldía Mayor de Bogotá, 2013, p. 9; Ramírez, 2017). Sin embargo, ese silencio es suplido por un discurso heteronormativo en el que, para la configuración de la identidad sexual y de género de los y las estudiantes, se realiza un proceso de diferenciación frente al trato que reciben o la asignación de roles en razón a las características genitales, con lo que se da a entender que existen lugares vetados para unos y otros, así como maneras únicas de ser hombres o mujeres (Ramírez y Martínez, 2013).

Quienes no corresponden a estos parámetros rígidos son sometidos a señalamientos, burlas y otras formas de violencia directa o indirecta. Para Hall (2003), la raíz de este tipo de violencia es la lógica del sistema heterocentrado y patriarcal, que propende por la mismidad y otredad; es decir, el mantenimiento de unos parámetros reconocidos como legítimos, deseables e irrefutables en cuanto a la identidad de los sujetos, que diferencia lo que se es a partir de lo que no se es. En los escenarios educativos la violencia en contra de los y las estudiantes LGBT, o percibidos como tal, es catalogada como bullying homofóbico, el cual tiene como función denunciar y reprimir a los y las estudiantes que son reconocidos fuera de la norma (una identidad), para conseguir que vuelvan a la norma.

Desde la anterior perspectiva, el bullying homofóbico es un dispositivo de normalización heterosexual, el cual se encuentra reforzado por las maneras en que tradicionalmente se ha constituido el discurso de la sexualidad. El dispositivo no debe entenderse como un ente o un sinónimo de máquina, puesto que no opera siempre de la misma forma para obtener el mismo resultado, sino 
que constantemente se reconfigura a sí mismo; de cierta manera, aprende como si estuviera dotado de inteligencia artificial y, por lo tanto, produce distintos tipos de subjetividades en cada momento histórico; esto es así porque no todos los individuos circulan por la totalidad de la red de discursos durante su existencia ni hacen el mismo recorrido $y$, fundamentalmente, porque los efectos de poder que produce un dispositivo no le dicen al sujeto que constituyen qué es lo que tiene que hacer, decir, pensar, ser a cada momento o en todo lugar (García, 2011).

\section{Discursos, prácticas y temáticas de maestros y maestras en primera infancia acerca de la diversidad sexual y de género}

Como se señaló antes, los discursos de los docentes fueron analizados desde el mapa o cartografía de referencias biográficas insertadas en el entorno sociocultural del que forman parte los autores, con el fin de establecer conceptos y creencias, a través de las expresiones utilizadas por estos al referirse a la diversidad sexual; con lo cual se identificaron los siguientes elementos.

El personal docente no tiene claridad en torno a conceptos tales como identidad sexual, identidad de género y orientación sexual, lo cual genera que en sus conceptualizaciones acerca de la diversidad sexual se caiga en continuos errores. Así mismo, se nota que en general existe una valoración negativa con respecto a la diversidad sexual y de género. Maestros y maestras consideran que es una deformación de la naturaleza humana; en la mayoría de los casos esta visión aparece reforzada por creencias religiosas, especialmente de tradición cristiana. La frecuencia de las palabras que utilizan los docentes para conceptualizar la diversidad sexual (figura 2) evidencia la prevalencia del discurso religioso. 
Figura 2. Nube de palabras que más utilizan los docentes para conceptualizar la diversidad sexual.

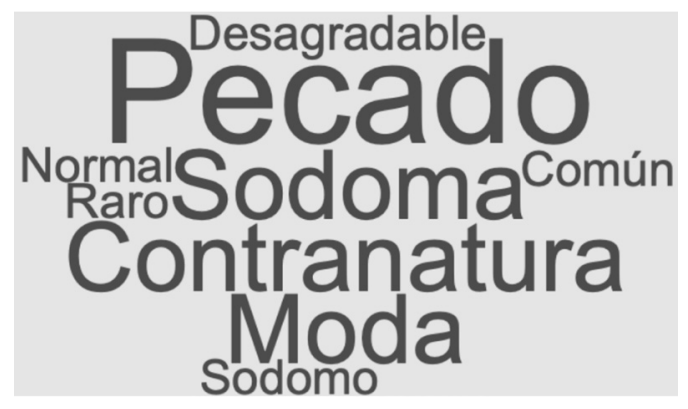

Fuente: construcción propia.

"Pecado" y "sodoma" son las palabras más utilizadas para hacer referencia a la homosexualidad; por su parte, al hablar de la transexualidad era más frecuente que se utilizaran las palabras "desorden mental" o "enfermedad", lo que constata la incidencia de los discursos tradicionales de la religión y la medicina en la manera de entender la diversidad sexual, tal y como lo explican autores como Ramírez (2019) y Di Segni (2013). Aunque menos frecuentes, también se utilizaron palabras como "común", "normal" y "moda", lo que podría indicar que algunos reconocen el género y la sexualidad como una construcción sexual.

Desde las características demográficas, no se logra establecer una diferencia significativa relacionada con el sexo y la edad de los participantes; diferencia que sí se nota al momento de analizar sus perfiles de formación: los maestros y maestras con mayor nivel académico fueron quienes moralizaron menos lo relacionado a la orientación sexual de los estudiantes; sin embargo, en lo relacionado a la transexualidad las visiones patologizantes son comunes a todos los niveles de formación.

Otro elemento significativo que emergió de los diálogos con los docentes es la idea de que existen culpables de la orientación sexual que asumen los niños y las niñas; se responsabiliza en primera instancia a los medios de comunicación que hacen cada vez más frecuente la presencia de "personas así": "muchos de los artistas dicen que son gais y eso hace que los chinos, que no tienen perso- 
nalidad, quieran imitarlos" (comunicación directa). También se responsabiliza a los cuidadores, especialmente a las madres, pues se considera que "si un machito es criado con muchos mimos por la mamá, nunca va a aprender a ser hombre; los niños deben aprender del papá a ser hombres y las niñas a ser mujeres al lado de la mamá" (comunicación directa).

Al buscar responsables de la manera en que construyen la sexualidad niños y niñas LGBT, se reconoce que esta es una construcción social, pero no así la heterosexualidad. Se asume que la heterosexualidad es una realidad natural porque conduce a la procreación; sin embargo, al momento de preguntar acerca de la finalidad de las relaciones sexuales en general hay un consenso en reconocer la dimensión del placer — sexualidad recreativa-; de hecho, se asume la postura generalizada de que las parejas heterosexuales no deberían tener tantos hijos y se ve como positivo el hecho que algunas parejas jóvenes tomen la decisión de no ser padres.

Frente a las prácticas que los docentes tienen en el abordaje de la diversidad sexual, se analizaron tres elementos, a saber: 1) experiencias en el quehacer docente, 2) experiencias personales y 3 ) experiencias ajenas (tabla 1). Frente al primer elemento se identificaron tres tipos de actitudes y acciones: positivas, indiferentes y negativas, tal como se expone en la tabla 2, para estratificas las actitudes y acciones en tres grupos característicos.

Tabla 2. Actitudes y acciones relacionadas con experiencias en el quehacer docente.

\begin{tabular}{|l|l|l|}
\hline \multicolumn{1}{|c|}{ Positivas } & \multicolumn{1}{|c|}{ Indiferentes } & \multicolumn{1}{c|}{ Negativas } \\
\hline $\begin{array}{l}\text { A los niños y las niñas } \\
\text { identificados como LGBT se } \\
\text { les brinda un acompañamiento } \\
\text { especial para que no sean } \\
\text { víctimas de las burlas de sus } \\
\text { otros compañeros. }\end{array}$ & $\begin{array}{l}\text { La diversidad sexual y de } \\
\text { género no es incumbencia de } \\
\text { la escuela; en el aula se tienen } \\
\text { estudiantes, la responsabilidad } \\
\text { docente es desarrollar los } \\
\text { temas del currículo. }\end{array}$ & $\begin{array}{l}\text { La homosexualidad no se } \\
\text { puede permitir en la escuela. } \\
\text { Los estudiantes percibidos } \\
\text { con una orientación sexual o } \\
\text { identidad de género diversa } \\
\text { deben ser intervenidos para } \\
\text { ayudarles a superar esa } \\
\text { deformación. }\end{array}$ \\
\hline
\end{tabular}

Fuente: clasificación propia de los autores a partir de los diarios de campo. 
Algunos docentes, aun sin tener un abordaje claro de la diversidad sexual, tienen una apuesta por la protección de los niños y niñas percibidos como LGBT; tratan de tener control frente a dónde están, con quién están y de esa manera evitar que sus compañeros los puedan agredir. No se lograron identificar prácticas que fueran más allá del cuidado para evitar agresiones por parte de sus compañeros. Un importante porcentaje de los docentes prefieren mantenerse al margen del tema y consideran que frente a los temas de la sexualidad es mejor estar distanciados para "evitar problemas"; la postura se justifica en la afirmación: "entre menos se sepa de los estudiantes menos problemas hay que enfrentar" (comunicación directa).

Otras actitudes que se identificaron corresponden a algunas que se clasificaron como negativas y se caracterizaban por:

- Considerar que los estudiantes LGBT tiene que ser "arreglados".

- Legitimar las prácticas de violencia de los compañeros para que los estudiantes LGBT se comporten normal.

- Denunciar las prácticas "anormales" de los estudiantes con los padres de familia para que ellos tomen cartas en el asunto.

Lo anterior se puede ilustrar con la siguiente narración:

Un día Fernandito llegó llorando al salón porque sus compañeros le tiraron el jugo del refrigerio encima mientas le cantaban "Fernandito mariquita en vez de pantalón se tiene que poner faldita". Cuando lo vi, le ayudé a limpiarse y le dije: "usted por que no trata de comportarse más como sus otros compañeros, juegue fútbol con ellos. Usted tiene que aprender a ser más hombrecito, yo no puedo andar cuidándolo, deje de ser nena y no llore más", ¡mmm! [silencio] en el fondo, yo sabía que lo que le hacían ahora sus compañeros le iba a ayudar al pelao más 
adelante, porque los que son como él no la llevan fácil.

(Diario de campo 3).

La docente considera que Fernandito es quien está mal y que debe cambiar su comportamiento, porque de lo contrario generaría más violencia, que a futuro podría ser peor de la que estaba sufriendo en ese momento. De esa manera se legitima la violencia de sus compañeros; Fernando, que espera encontrar el apoyo de su maestra, es revictimizado, pues esta lo responsabiliza de la agresión que sufrió y lo carga de la responsabilidad al momento de hacerle ver que mientras él no se comporte como se esperaría al ser un hombre, se seguirían presentando estas violencias, frente a las que ella no intervendría.

Por último, no se logró reconocer ninguna experiencia en la que los docentes incorporaran entre los temas del currículo la diversidad sexual y de género. Lo que ratifica lo ya dicho por Peixoto, Fonseca, Almeida y Almeida (2012) acerca de cómo en el sistema educativo rige la ley del silencio, pese a que hace mucho que es cuestionada por los movimientos sociales y académicos (p. 146).

\section{Límites y posibilidades para la consolidación de una educación inclusiva}

Descrito lo anterior, se pueden establecer los siguientes elementos como límites para la consolidación de una educación inclusiva que reconozca, respete y valores a los estudiantes LGBT:

1. La falta de información que tienen los maestros y maestras acerca de la diversidad sexual, lo que no les permite tener una conceptualización más allá de los prejuicios estereotipados.

2. La perspectiva ideológica desde la cual algunos docentes asumen la diversidad sexual, con lo cual desconocen los 
marcos normativos que garantizan los derechos de las personas LGBT.

3. La autoconcepción del quehacer docente que tienen algunos maestros y maestras, que los mantienen aún en lógicas meramente cognitivas, en las cuales se entiende el proceso pedagógico como un acto para instruir a niños y niñas en áreas específicas del currículo y no como una posibilidad para la transformación, el reconocimiento de las diferencias y la convivencia pacífica.

4. La formación de algunos docentes de primera infancia, quienes en un alto porcentaje no han cursado programas posgraduales en el área específica o en educación, y no han recibido formación especializada para la atención de la diversidad sexual y de género en el aula.

Estas condiciones particulares dificultan la elaboración de un ejercicio pedagógico inclusivo, no por las condiciones complejas que implica, sino por los conceptos que circulan entre los docentes, que por lo general no cuentan con sustento biológico, pedagógico o legal; sino que parten de estereotipos promovidos por lógicas y discursos hegemónicos y hegemonizantes (González Gavaldón, 1999); estas dinámicas sociales se sitúan en la escuela y modifican las percepciones de maestros y maestras frente a la inclusión de estudiantes sexualmente diversos.

En cuanto a las posibilidades, se logra identificar que:

1. La educación avanzada de maestros y maestras contribuye a la superación de prejuicios y estereotipos en la manera de asumir la diversidad sexual y de género.

2. Existen actitudes positivas que buscan salvaguardar y proteger la integridad de niños y niñas que son percibidos como LGBT, que, si bien no son suficientes, sí pueden direccionarse para generar experiencias exitosas 
respecto al reconocimiento, respeto y valoración de la diversidad sexual y de género en la escuela.

3. Las actitudes indiferentes pueden modificarse con procesos formativos que ayuden a reorientar el quehacer docente a partir del análisis de las actividades rectoras en la educación para la primera infancia, especialmente en lo relacionado con la exploración del medio, que apunta al reconocimiento del entorno social en que se desarrollan los niños y las niñas.

\section{A manera de conclusión}

Si bien el presente estudio no tiene el alcance para determinar los límites y las posibilidades de la educación inclusiva para estudiantes con orientaciones sexuales e identidades de género diversas, sí permite formarse una idea de lo que concierne a los discursos y prácticas que algunos maestros de primera infancia tienen al respecto de este tema; además de que se presenta una caracterización de los elementos generales que circulan entre los docentes y que se refieren a los estudiantes que se perciben como sexualmente diversos.

A partir de la metodología asumida en el trabajo de investigación, la lógica narrativa está guiada por los investigadores, expertos en el tema de la inclusión educativa de estudiantes con orientaciones sexuales e identidades de género diversas; ello generó como resultado una autoconciencia acerca de la realidad social y del contexto histórico-cultural en el que se realizó el ejercicio investigativo, más allá de los propios instrumentos de observación y diarios de campo.

Aunque los avances en materia de garantía de derechos de estudiantes LGBT son amplios en la formulación de las leyes y el desarrollo de políticas públicas, estas no han tenido un importante efecto en los sistemas educativos, y los docentes siguen con ac- 
tuaciones más desde su "percepción" y "creencia" al respecto que desde la perspectiva de derechos, ni como respuesta a las tipificaciones en la legislación colombiana.

Es necesario realizar procesos de formación avanzados destinados a profundizar en torno a las perspectivas teóricas, jurídicas y pedagógicas relacionadas con la atención de estudiantes LGBT; por ello, esta propuesta, incluidos sus hallazgos, se constituye en un elemento importante para el planteamiento de estrategias que lleven a la concientización y a fortalecer los conocimiento de los maestros y maestras tanto en lo legal como en lo biológico, personal y social, a fin de que las pedagogías incluyentes partan del reconocimiento, respeto y valoración de las diferencias a todo nivel.

\section{Referencias bibliográficas}

APA (American Psychological Association) (2002). Respuestas a sus preguntas: para una mejor comprensión de la orientación sexual y la homosexualidad. Recuperado de https://www.apa.org/topics/lgbt/ answers-questions-so-spanish.pdf

Alcaldía Mayor de Bogotá (2013). Diagnóstico de situación de niñas, niños y adolescentes con orientaciones sexuales o identidades de género no normativas. Imprenta Distrital. Bogotá, Colombia

Armstrong, E. A. y Crage, S. M. (2006). Movements and memory: The making of the Stonewall Myth. American Sociological Review, 71(5), 724-751. DOI: https://doi.org/10.1177/000312240607100502

Atkinson, P. (2013). Ethnographic writing, the avant-garde and a failure of nerve. International Review of Qualitative Research, 6(1), 19-35.

Banguero Camacho, V. (2007). El horizonte de sentido. Desafíos de las disciplinas de las ciencias sociales: organización de conocimiento desde la investigación. Entramado, 3(1), 8-13. 
BBC (6 de septiembre de 2018). India: el histórico fallo que despenaliza la homosexualidad después de 157 años. Recuperado de https://www. bbc.com/mundo/noticias-internacional-45431634

Blanco, M. (2012). Autoetnografía: una forma narrativa de generación de conocimientos. Andamios, 9(19), 49-74. Recuperado de http:// www.scielo.org.mx/scielo.php?script=sci_arttext \&pid=S1870 $00632012000200004 \& \operatorname{lng}=e s \&$ tlng=es

Di Segni, S. (2013). Sexología, género y heteronormatividad. Recuperado de http://jornadascinig.fahce.unlp.edu.ar/iii-2013/actas-2013/DiSegni. pdf

El Espectador (8 de agosto de 2016). ¿Qué hay detrás de falsas cartillas sobre educación sexual que achacan al Mineducación? Recuperado de https://www.elespectador.com/noticias/educacion/hay-detras-defalsas-cartillas-sobre-educacion-sexual-a-articulo-647998

Fonseca Hernández, C. y Quintero Soto, M. (2009). La teoría queer: la de-construcción de las sexualidades periféricas. Sociológica, 69(24), 4360.

Foucault, M. (2007). Historia de la sexualidad. Ciudad de México: Siglo XXI.

Garcia, L. (2011). ¿Qué es un dispositivo?: Foucault, Deleuze, Agamben. Revista A parte Rei, 74. Marzo. Revista electrónica de filosofía, disponible en http://serbal.pntic. mec.es/AParteRei/fanlo74.pdf

García Gómez, S. (2014). Diario de campo [reseña del libro Diario de campo de R. Izquierdo Chaparro (2013)]. Pedagogía Social: Revista Interuniversitaria, 24, 287-289. Recuperado de https://recyt.fecyt.es/ index.php/PSRI/article/view/37149

González Gavaldón, B. (1999). Los estereotipos como factor de socialización en el género. Comunicar, (12), 79-88. Recuperado de https://www.redalyc.org/pdf/158/15801212.pdf 
Guerrero, J. (2014). El valor de la auto-etnografía como fuente para la investigación social: del método a la narrativa. Azarbe: Revista Internacional de Trabajo Social y Bienestar, 3, 237-242.

Guerrero, J. (2016). Autoetnografía y práctica social transformativa. En: J. E. Martínez y B. de Maya (Eds). Perspectivas interdisciplinares en el estudio de la cultura y la sociedad. Ecuador: Universidad Miguel Hernández.

Hall, S. (2003) ¿Quién necesita "identidad? Cuestiones de identidad cultural. Buenos Aires: Amorrortu.

Hayano, D. (1979). Auto-ethnography: Paradigms, problems and prospects. Human Organization, 38(1), 99-104.

Heider, K. G. (1975). What do people do? Dani auto-ethnography. Journal of Anthropological Research, 31(1), 3-17.

Mena-López, M. y Ramírez, F. (2018). Falacias discursivas en torno a la ideología de género. Ex AEquo, 37, 19-31. DOI: https://doi.org/10.22355/ exaequo.2018.37.02

Núñez, G. (2016). ¿Qué es la diversidad sexual? Ciudad de México: Ariel.

Panourgia, N. (2000). Auto/ethnography: Rewriting the self and the social. American Ethnologist, 27(2), 551-553. DOI: https://doi. org/10.1525/ae.2000.27.2.551

Peixoto, J., Fonseca, L., Almeida, S. y Almeida, L. (2012). Escuela y diversidad sexual - ¿que realidad? Educação em Revista, 28(03), 143158.

Ramírez, F. (2017). Inclusión educativa de estudiantes sexualmente diversos en Colombia, periodo 2000-2015 (tesis de doctorado). Universidad Santo Tomás, Bogotá. Recuperado de http://repository. usta.edu.co/handle/11634/4074 
Ramírez, F. (2019). Democracia, sexualidad y religión, el caso colombiano. En G. Careaga (coord.), Sexualidad, religión y democracia en América Latina (pp. 119-140). Ciudad de México: Fundación Arcoíris por el Respeto a la Diversidad Sexual.

Ramírez, F. y Martínez, A. (2013). El género y su representación social en niños y niñas de una escuela de Boyacá. Pre-til: Investigar para Hacer Ciudad, 29(11), 17-30.

Semana (9 de septiembre de 2016). Ideología de género, el caballo de batalla del No al plebiscito. Semana [en línea]. Recuperado de https:// www.semana.com/nacion/articulo/ideologia-de-genero-el-caballo-debatalla-del-no-al-plebiscito/493093

Urra, E., Muñoz, A. y Peña, J. (2013). El análisis del discurso como perspectiva metodológica para investigadores de salud. Enfermería Universitaria, 10(2), 50-57. DOI: https://doi.org/10.1016/S16657063(13)72629-0

Vilá, E. (2016). La "ideología de género": la caza vaticana a un fantasma. Iglesia Viva: Revista de Pensamiento Cristiano, 267, 75-88. Recuperado de https://dialnet.unirioja.es/servlet/articulo?codigo $=5684764$ 